\title{
Quantification of inflammatory bowel disease activity using technetium-99m HMPAO labelled leucocyte single photon emission computerised tomography (SPECT)
}

Departments of Biochemical Medicine M J Weldon

J D Maxwell

Radiology

J Gane

A E A Joseph

Medical Physics

A M Masoomi

A J Britten

and Histopathology

C J Finlayson

St George's Hospital and Medical School,

London

Correspondence to:

Dr M J Weldon,

Department of Biochemical

Medicine, St George's

Hospital and Medical

School, Cranmer Terrace,

London SW 17 ORE.

Accepted for publication

27 May 1994

M J Weldon, A M Masoomi, A J Britten, J Gane, C J Finlayson, A E A Joseph, J D Maxwell

\begin{abstract}
Technetium-99m HMPAO labelled white cell bowel uptake was quantified in 23 patients with suspected colitis by means of the novel technique of single photon emission computerised tomography (SPECT) using a standard gammacamera. Uptake in colon segments was quantified on transaxial images and expressed as a fraction of marrow uptake (SPECT score). Segmental histological disease activity was assessed at colonoscopy (20) or at surgery (3). Segmental histology score correlated with SPECT score $r=0.79 \quad(p<0.001)$. Overall SPECT score for all affected segments correlated with Crohn's disease activity index (CDAI) $r=0.66, p=0.001$ and with laboratory activity indices; erythrocyte sedimentation rate $r=0.44$, $p=0.03$, C reactive protein $r=0.38$, $p=0.05$, and albumin $r=-0.46, p=0.03$. Small bowel SPECT score in 13 patients correlated with CDAI $r=0.65, p<0.01$ but not with erythrocyte sedimentation rate or $\mathbf{C}$ reactive protein. Five patients with positive small bowel white cell uptake had normal $C$ reactive protein and erythrocyte sedimentation rate. Overall SPECT score for disease at all sites correlated with both CDAI and with laboratory indices of inflammation. ${ }^{99 m}$ Tc HMPAO SPECT provides non-invasive and accurate quantification of inflammatory bowel disease activity in both large and small bowel and may be useful in the objective evaluation of treatment for inflammatory bowel disease.

(Gut 1995; 36: 243-250)
\end{abstract}

Keywords: inflammatory bowel disease, technetium-99m, single photon emission computerised tomography.

As neutrophils migrate to areas of acute inflammation, bowel scintigraphy using radiolabelled leucocytes has been used in the assessment of disease extent and activity in inflammatory bowel disease. Early gammacamera studies using ${ }^{111}$ Indium showed that bowel scans accurately assessed disease extent when compared with radiology, endoscopy, and histology. ${ }^{1}$ Measurement of faecal excretion of ${ }^{111}$ Indium labelled leucocytes over four days was also shown to reliably assess disease activity in Crohn's disease. ${ }^{2}$ Drawbacks of ${ }^{111}$ Indium scanning, however, include the isotope's long half life (67 hours), comparatively high radiation dose, and the need for granulocyte purification for optimal quantification. Furthermore, faecal collection requires patient cooperation and is unpopular with staff.

More recently, technetium-99m hexamethyl propylene amine oxime (HMPAO), originally introduced for regional cerebral blood flow imaging, has been found to selectively label granulocytes in mixed leucocyte suspensions. ${ }^{3}$ Experience with ${ }^{99 \mathrm{~m}} \mathrm{Tc}$ HMPAO labelled white cells in the imaging of inflammatory bowel disease has been encouraging. ${ }^{4}$ It has important advantages over ${ }^{111}$ Indium in imaging intestinal inflammation including shorter image acquisition times, improved image quality, and reduced radiation dose. In routine clinical practice, visual inspection of the degree of uptake in bowel, in comparison with the uptake in marrow, liver, and spleen (in order of increasing intensity) can provide some information to quantify disease activity. However, such methods are subjective. ${ }^{5}$ Moreover, they suffer from the problem of overlapping activity in other organs, particularly bone marrow.

In view of the difficulties with quantification using planar imaging, we have applied an alternative gammacamera imaging approach; SPECT (single photon emission computerised tomography). This technique has already proved useful in quantitative studies on blood flow in brain and heart. ${ }^{6}$ We have applied this technique to the assessment of disease activity in ulcerative colitis and Crohn's colitis, using transaxial abdominal slice images in a manner analagous to $x$ ray computed tomography. We have compared the SPECT derived isotope uptake score for each segment of involved bowel with the degree of inflammation in each segment assessed histologically. We also report our preliminary findings in the assessment of small bowel Crohn's disease.

\section{Methods}

PATIENTS

Twenty three patients, 14 female and nine male, mean age 36 (range 22-74) with 
suspected active colitis were investigated by both ${ }^{99 \mathrm{~m}}$ Tc HMPAO SPECT and histological examination. Eight were known to have ulcerative colitis and six were known to have Crohn's disease, based on standard endoscopic, radiological, and histological criteria. Known distribution of disease, based on previous colonoscopy or barium radiology was pancolitis in four, subtotal colitis in one, left sided colitis in five, distal colitis in two, and small bowel disease alone in two. Mean duration of disease was 3.2 years (range one month to 12 years). The remaining nine were new patients presenting to the gastroenterology clinic with diarrhoea or abdominal pain. Final diagnoses in these patients, based on all available criteria were - two ulcerative colitis, six Crohn's disease, and one irritable bowel syndrome.

Thirteen further patients were investigated for small bowel inflammation using ${ }^{99 \mathrm{~m}} \mathrm{Tc}$ HMPAO SPECT. Ten were female and three male. Twelve were known to have small bowel Crohn's disease on the basis of previous barium radiology. One of these had undergone previous strictureplasty, and four had a history of previous right hemicolectomy. Mean age was 32 (range 21-52). Seven were receiving oral corticosteroid treatment and one was also taking azathioprine.

The study was approved by the local research ethics committee.

\section{LEUCOCYTE LABELLING}

A standard protocol was used to label mixed white cells. ${ }^{7}$ Briefly, $102 \mathrm{ml}$ of venous blood were taken into $18 \mathrm{ml}$ of acid citrate dextrose. Hespan was used as a sedimenting agent. Blood was left to sediment for 30-60 minutes. The supernatant, containing leucocyte rich, platelet rich cells was centrifuged at $150 \mathrm{~g}$ for five minutes to give a pellet of mixed white cells and a supernatant of platelet rich plasma, which was removed. The pellet was resuspended in $1 \mathrm{ml}$ of cell free plasma, obtained by centrifugation of $20 \mathrm{ml}$ of blood at $2000 \mathrm{~g}$ for 10 minutes. Four $\mathrm{ml}$ of ${ }^{99 \mathrm{~m}} \mathrm{Tc}$ exametazime solution was added and incubated for 10 minutes. After incubation, $10 \mathrm{ml}$ of plasma was added and the tube was centrifuged at $150 \mathrm{~g}$ for five minutes. The pellet was resuspended in $5 \mathrm{ml}$ of plasma ready for reinjection. Two hundred $\mathrm{MBq} \pm 10 \mathrm{MBq}$

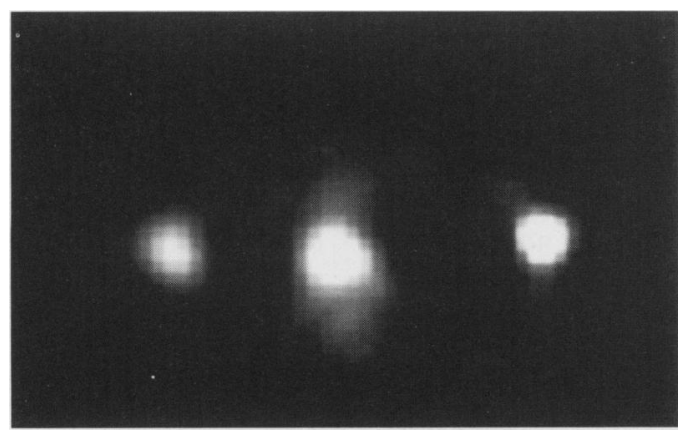

Figure 1: Transaxial mid abdominal slice image from patient with total colitis showing ascending (left) and descending (right) colon and bone marrow activity (centre). of ${ }^{99 \mathrm{~m}} \mathrm{Tc}$ HMPAO labelled white cells in plasma were injected.

IMAGING AND CALCULATION OF SPECT SCORE Anterior and pelvic view planar abdominal images were acquired 60 minutes after reinjection of radiolabelled leucocytes to show the extent of disease. Pelvic outlet views were required to image the rectum. A low energy general purpose collimator (Elscint APC-34) was used for all imaging with an Elscint SP6 gammacamera, recording for 180 seconds per view into $256 \times 256$ image matrices.

At 1.5-2 hours after reinjection of cells, SPECT was performed. A $360^{\circ}$ circular orbit was used of 30 minutes duration with continuous motion and $6^{\circ}$ per view. An image matrix of $64 \times 64$ pixels was used with a pixel size of $8.4 \mathrm{~mm}$.

After data acquisition, images were processed as follows: a Ramp reconstruction filter with Hanning window was used to produce the transaxial images. Correction for attenuation was made using the Chang technique, ${ }^{8}$ with an attenuation coefficient of $0.12\left(\mathrm{~cm}^{-1}\right)$. Figure 1 shows the transaxial abdominal slices of $16.8 \mathrm{~mm}$ thickness that were reconstructed.

Previous studies have shown that activity in colon segments can be identified on transaxial SPECT slices. ${ }^{9}$ The following five segments: ascending colon, transverse colon, descending colon, sigmoid colon, and rectum were identified for analysis. In small bowel disease, simple planar quantification of this image might overestimate the bowel activity as there is often underlying pelvic marrow activity. We analysed pelvic transaxial SPECT slices to separate small bowel activity from pelvic activity potentially permitting small bowel uptake quantification.

The maximum counts per pixel in each of these segments were measured using region of interest analysis of serial slices to obtain the maximum value for each bowel segment. The total activity in each segment was not measured. The SPECT segment score was expressed as a fraction of marrow activity, obtained by averaging the maximum counts in five transaxial slices of lumbar spine. Preliminary studies showed that using this method, interobserver variation was $<5 \%$. All of the SPECT processing was carried out by one observer (MJW). With regard to the time taken for the examination, the initial planar images took six minutes for anterior and inferior views, the SPECT acquisition 30 minutes, and the subsequent processing and calculation about one hour giving a total time of 1 hour 36 minutes.

CLINICAL ASSESSMENT OF DISEASE ACTIVITY Laboratory assessments of disease activity were made using erythrocyte sedimentation rate, $\mathrm{C}$ reactive protein, platelet count, and serum albumin. Histological examination was taken as the gold standard measure of mucosal disease activity. This was obtained at 
TABLE I Histology scoring system for each colonic segment

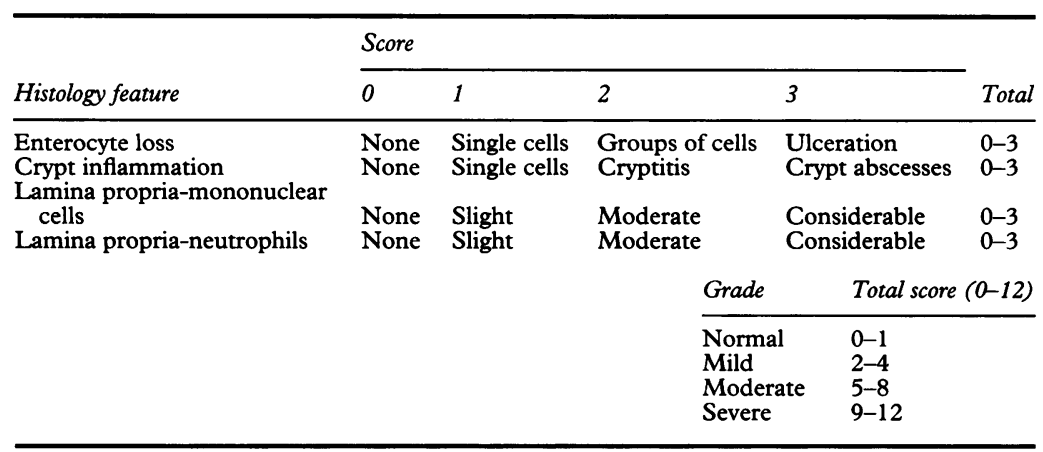

This histological scoring system was used for biopsy specimens obtained at colonoscopy or surgery. A final grade of normal, mild, moderate or severe disease activity was obtained.

colonoscopy in 20 patients and at resection in three patients. (Resection was within eight, seven, and two days of SPECT.) Colonoscopy or resection was within 10 days of SPECT in all cases with a median separation of three days.

Biopsy specimens were obtained from the same five bowel segments as used for SPECT. The histopathologist graded the colonic specimens for severity of inflammation using a standard scoring system, which has been used in previous studies ${ }^{1}$ (see Table I), and was unaware of clinical or colonoscopic findings. The same scoring system was used for resection specimens.

Derivation of CDAI (Crohn's disease activity index) - this empirical disease activity scoring system was used for both Crohn's disease and

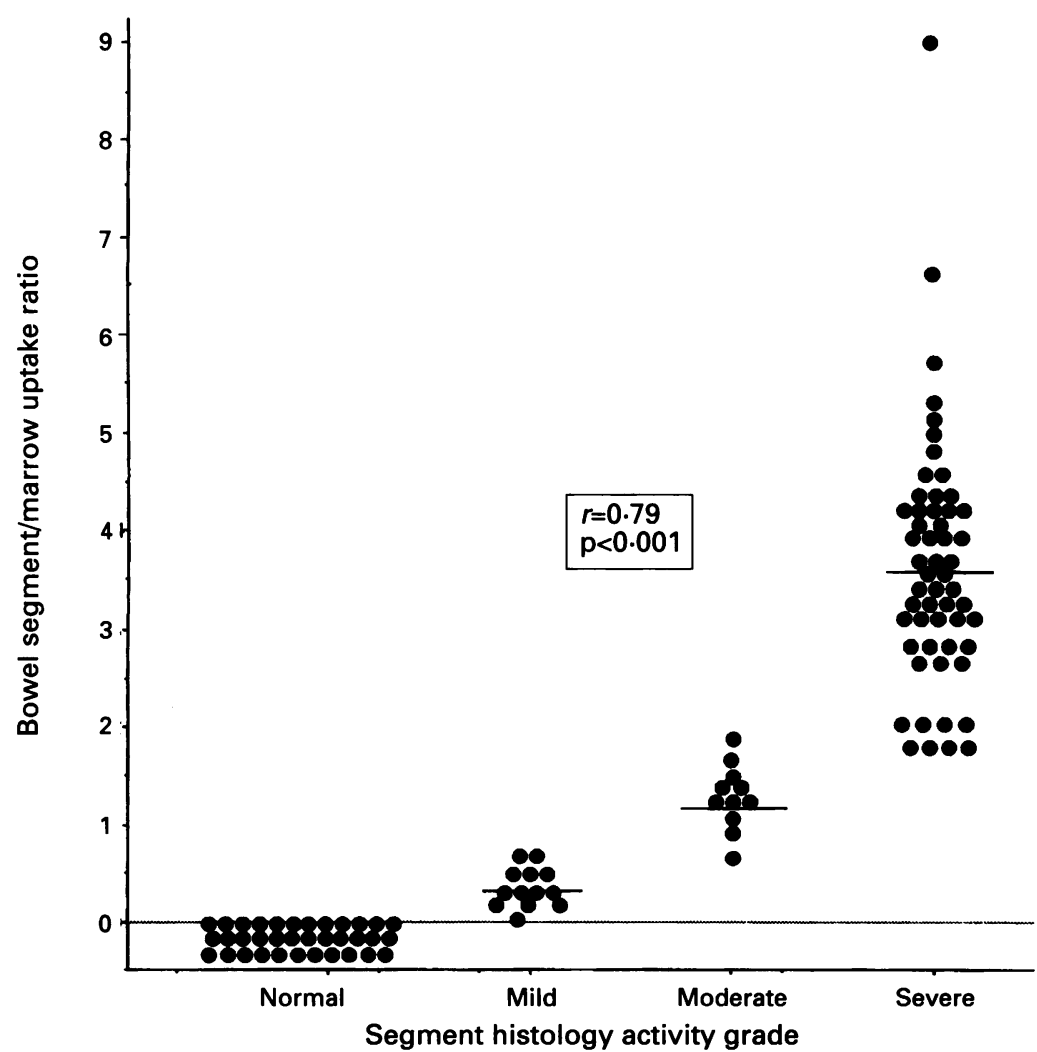

Figure 2: There is a correlation of $\mathrm{r}=0.79 p<0.001$ between the SPECT score and histology disease activity grade for each colonic segment. The mean (SD) SPECT scores for normal; 0 , mild; $0.326(0.19)$, moderate; $1.11(0.36)$, and severe; $3.33(1.84)$ histology grades were significantly different, $p<0.001$ (Mann-Whitney $U$ test). Horizontal bars represent the mean. ulcerative colitis, using a diary card, recording symptoms over the week preceding assessment as has been used in previous studies of inflammatory bowel disease. ${ }^{1}$ For both Crohn's disease and ulcerative colitis, the coefficients originally designed for Crohn's disease were used. The disease activity ranged from 10 to 544. The three patients requiring colectomy had CDAI values of 464, 467, and 544 . (Active disease was defined as CDAI $>150 .{ }^{10}$ )

\section{STATISTICS}

Correlations between the SPECT score and CDAI, and laboratory measurements were tested using Spearman's rank correlation coefficient where $r_{\mathrm{s}}$ denotes Spearman's coefficient and $\mathrm{p}$, the associated probability. Comparisons between discontinuous variables were made using the Mann-Whitney U test.

\section{Results}

NEGATIVE ${ }^{99} \mathrm{~m}_{\text {TC }}$ HMPAO LARGE BOWEL SCINTIGRAMS

The planar one hour image was normal in seven patients. All of these patients also had normal SPECT with resulting SPECT scores of zero. Six of seven of these patients also had normal colonoscopy with no evidence of active colitis on histological examination. The remaining patient with previously clinically active ulcerative colitis had normal colonoscopy and was clinically asymptomatic receiving only maintenance mesalazine treatment but there was evidence of mild inflammation in the sigmoid colon on biopsy, of questionable significance.

POSITIVE ${ }^{99} \mathrm{~m}$ TC HMPAO LARGE BOWEL SCINTIGRAMS

The planar image was positive in 16 patients. In all of these cases, it was possible to visualise the segments seen on planar images on the SPECT transaxial slices as seen in Fig 1, and to perform quantification. Figure 2 shows that there was a close correlation of $r=0.79$ between the SPECT score for a given segment of bowel and the histological activity grade. There was also good separation of histology grades according to the SPECT score that was statistically significant.

An overall disease activity score could be derived by summating the segment SPECT scores for each patient. This score correlated with the CDAI $r=0.66$ as well as with laboratory measurements; erythrocyte sedimentation rate $r=0.44, \mathrm{C}$ reactive protein $r=0.38$, albu$\min r=0.46$ (Table II).

A commercial computer program (Elscint A3D) was used to create a pseudo 3D display of the colonic image in three dimensional form as a rotating cine image. Using such displays, the spatial position of the colon could be shown (Fig 3).

Three patients required urgent resection because of failure to respond to medical treatment. In all of these cases the histological 
TABLE II Correlation matrix between SPECT scan score and other measures of disease activity

\begin{tabular}{|c|c|c|c|c|c|c|}
\hline \multirow{2}{*}{$\begin{array}{l}\text { Index } \\
\text { correlation }\end{array}$} & \multicolumn{2}{|l|}{ Colon } & \multicolumn{2}{|c|}{ Small bowel } & \multicolumn{2}{|l|}{ All sites } \\
\hline & $r_{s}$ & $p$ & $r_{s}$ & $p$ & $r_{s}$ & $p$ \\
\hline Histology & 0.79 & $\mathrm{p}<0.001$ & NA & NA & NA & NA \\
\hline CDAI & 0.66 & $\mathrm{p}=0.001$ & 0.65 & $\mathrm{p}=0.01$ & 0.69 & $\mathrm{p}=0.001$ \\
\hline ESR & 0.44 & $\mathrm{p}=0.03$ & $0 \cdot 1$ & NS & 0.45 & $\mathrm{p}<0.001$ \\
\hline Albumin & -0.46 & $\mathrm{p}=0.03$ & -0.4 & $p=0.04$ & -0.3 & $\mathrm{p}=0.1$ \\
\hline Platelets & 0.44 & $\mathrm{p}=0.03$ & $0 \cdot 1$ & NS & 0.34 & $p=0.1$ \\
\hline CRP & 0.38 & $\mathrm{p}=0.05$ & $0 \cdot 1$ & NS & $0 \cdot 34$ & $\mathrm{p}=0.03$ \\
\hline
\end{tabular}

$\mathrm{ESR}=$ erythrocyte sedimentation rate; $\mathrm{CRP}=\mathrm{C}$ reactive protein.

Correlation matrix between SPECT scan overall score and other measures of disease activity.

The best correlation was with histology; the most direct measure of bowel inflammation.

NA = not applicable. small bowel inflammation had negative scans. The first patient had a CDAI score greater than 150 with a negative SPECT scan, which was caused by a fibrous stricture shown on barium meal causing abdominal pain. Erythrocyte sedimentation rate and $\mathrm{C}$ reactive protein were normal in this patient, the second had irritable bowel syndrome on the basis of normal small bowel radiology, normal erythrocyte sedimentation rate, $\mathrm{C}$ reactive protein, and colonoscopy, and the third had a recent hemicolectomy for severe terminal ileal Crohn's disease and was found to have no evidence of recurrent disease on the basis of colonoscopy including ileoscopy. Combining patients with disease at all sites, correlations were found between overall bowel SPECT score and erythrocyte sedimentation rate $r=0.45$; C reactive protein $r=0 \cdot 34$, and CDAI $r=0.69$ (Table II). Figure 5 shows the correlation between CDAI and overall SPECT score for all patients.

In 10 patients, the planar scan showed active small bowel disease. In all of these patients, the pelvic SPECT slice showed small bowel activity, clearly separated from pelvic activity (see Fig 4), which could also be quantified and expressed as a ratio of marrow activity.

There was a good correlation between CDAI and small bowel SPECT score of $r=0 \cdot 65$, (Table II). Five patients with definite small bowel inflammation on both planar and SPECT imaging also had normal erythrocyte sedimentation rate and $\mathrm{C}$ reactive protein. No direct correlation was therefore seen between small bowel SPECT score and $C$ reactive protein or erythrocyte sedimentation rate. Three of the patients investigated for suspected
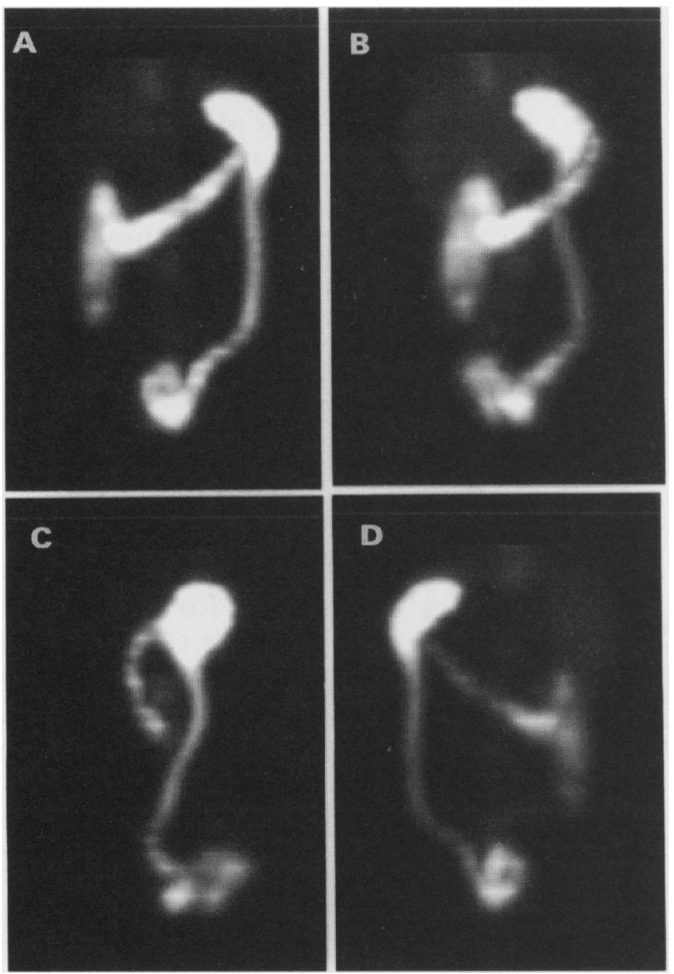

Figure 3: Example of 3D SPECT reconstructed image from a patient with pancolitis. Frozen frames from the rotating image at (A) anterior, $(B)$ oblique, (C) lateral, and (D) posterior viewpoints are seen.

\section{Discussion}

Measurement of inflammatory bowel disease activity is important in the assessment of patients with ulcerative colitis and Crohn's disease. As these disorders are relapsing conditions, repeated assessments are often required. Many methods to assess disease activity have been proposed such as laboratory indices, for example, erythrocyte sedimentation rate and $C$ reactive protein and clinical scores, ${ }^{10-12}$ but they are limited by subjectivity, low sensitivity, ${ }^{13}$ and non-specificity. There is still no generally accepted indicator of disease activity in inflammatory bowel disease and none fulfils all the requirements of an ideal test - simple, inexpensive, safe, non-invasive, convenient, acceptable to patients and staff, objective, reliable, and amenable to serial measurement to permit the assessment of various therapeutic interventions.

Endoscopy is undoubtedly useful to directly visualise and biopsy diseased bowel but has some limitations; access to diseased bowel is not always possible because of stricturing or proximal small bowel disease; it is also undesirable to perform repeated examinations as this invasive procedure requires bowel preparation, may be uncomfortable, and is contraindicated in very active disease. A less invasive approach is therefore needed.

Although inflammatory bowel disease is a chronic disease with a predominantly mononuclear infiltrate, during a relapse, there is acute on chronic inflammation with neutrophil recruitment although the mechanism of neutrophil recruitment to the bowel in Crohn's disease and ulcerative colitis may differ. ${ }^{14}$ Previous workers have successfully used radiolabelled neutrophils to image inflammatory bowel disease. ${ }^{1}$ Initially, ${ }^{111}$ Indium labelled leucocytes were used to localise and estimate disease activity. ${ }^{115}$ This was achieved by measuring the faecal excretion of ${ }^{111}$ Indium labelled white cells over four days. ${ }^{2}$ Although this was initially claimed to be a gold standard parameter for disease activity in inflammatory 


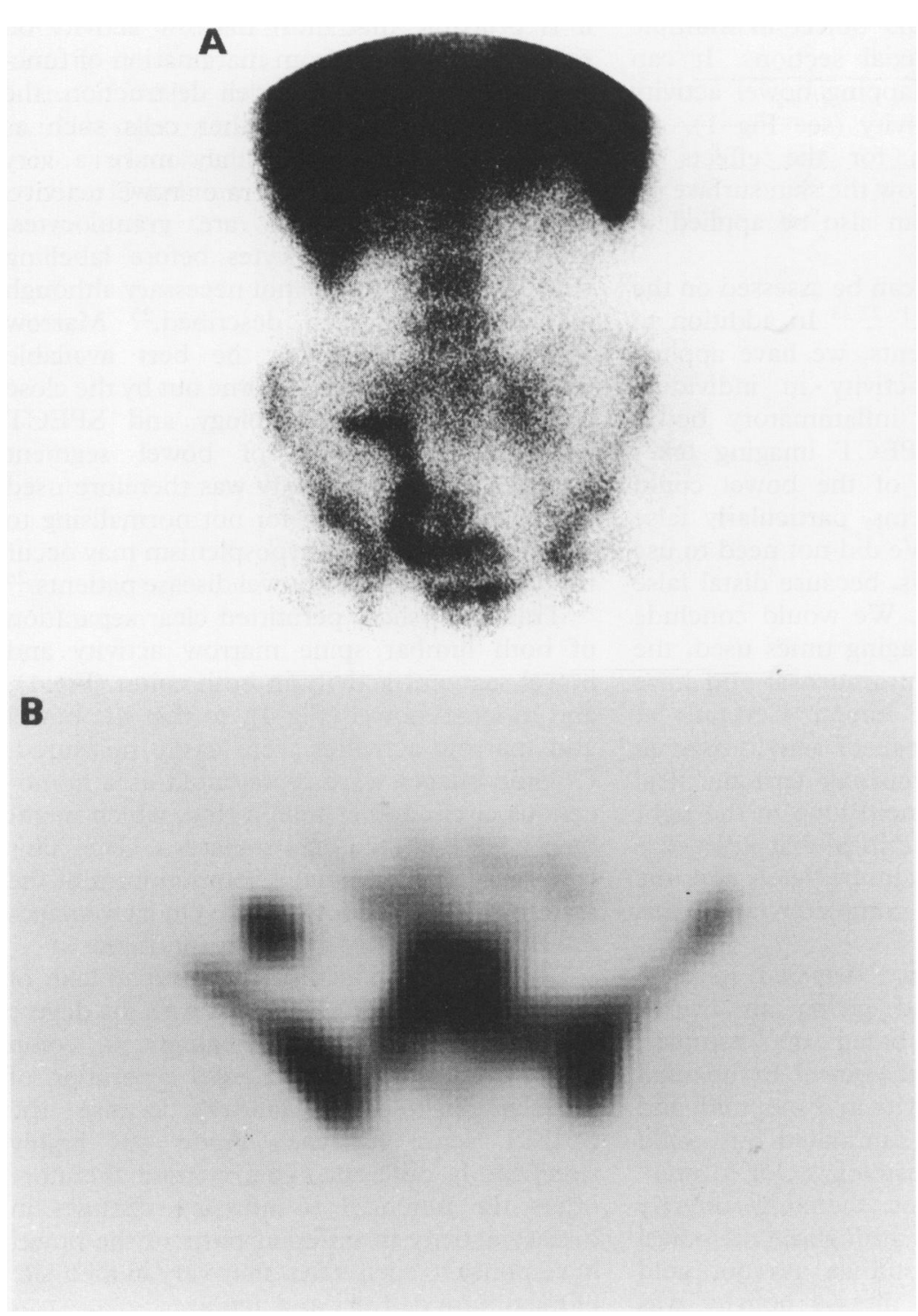

Figure 4: Example of $(A)$ planar image showing small bowel activity overlying pelvic bone activity and $(B)$ pelvic SPECT transaxial slice showing small bowel activity clearly separated from pelvic bone activity. high radiation dose limits repeated studies. Recently, total body quantification of residual ${ }^{111}$ Indium at four days after administration of ${ }^{111}$ Indium labelled granulocytes with derivation of bowel uptake by subtraction has been reported. ${ }^{16}$ While this avoids problems of faecal collection, the requirement to restudy the patient at a later date is inconvenient. Moreover, because of the superior quality images, reduced radiation dosimetry, and the short, simple cell labelling procedure, ${ }^{99 \mathrm{~m}}$ Tc HMPAO has replaced ${ }^{111}$ Indium as the isotope of choice for imaging acute intestinal inflammation. ${ }^{17}$ However, can ${ }^{99 \mathrm{~m}} \mathrm{Tc}$ HMPAO labelled white cells be used to assess disease activity?

\section{ASSESSMENT OF DISEASE ACTIVITY USING}

\section{VISUAL SCAN GRADING}

Faecal collection studies using ${ }^{99 m}$ Tc HMPAO labelled leucocytes are not possible because of its short half life and non-specific biliary excretion, which is often seen after four hours. ${ }^{18}$ Grading of bowel uptake of both ${ }^{111}$ Indium and ${ }^{99 \mathrm{~m}}$ Tc HMPAO by visual comparison with uptake in spleen, liver, and bone marrow has been described. ${ }^{15} 19$ The uptake intensity on scanning using ${ }^{111}$ Indium labelled granulocytes correlates closely with clinical disease activity indices ${ }^{20}$ and endoscopy with histology. ${ }^{1}$ This method, however, is rather crude and subjective and prone to high interobserver variation. ${ }^{5}$ This does not limit its clinical use but more objective quantification is needed for comparative studies. ${ }^{1}$

\section{ASSESSMENT OF DISEASE ACTIVITY USING COMPUTERISED PLANAR IMAGING}

The important factors that affect detection of bowel radioactivity counts using planar quantification techniques are: firstly, overlap of activity in bowel and other structures such as bone marrow (as seen in Fig 4A) and secondly, the changing depth of bowel below the skin surface causing variable attenuation of counts.

A recently described planar method entails subtraction of the average background activity derived from a series of previous abnormal scans. ${ }^{21}$ This method therefore results in an approximation of the overlap factor and indeed the resulting scan score was found to correlate well with various clinical and laboratory measures of disease activity, although it was not compared with histology. This method does not, however, compensate for variations in bowel depth. At present this technique also requires specific computer software, which is not yet widely available.

\section{ASSESSMENT OF DISEASE ACTIVITY USING} SPECT

SPECT is an imaging technique that measures the three dimensional distribution of counts. This is achieved by rotating the gammacamera around the patient, acquiring counts from a wide range of angles. It produces cross 
sectional images of a $3 \mathrm{D}$ object in multiple planes including transaxial sections. It can therefore separate overlapping bowel activity from bone marrow activity (see Fig 1). An approximate correction for the effects of variable bowel depth below the skin surface on such transaxial slices can also be applied to permit quantification.

The extent of disease can be assessed on the one hour planar image. ${ }^{1922} 23$ In addition to locating involved segments, we have applied SPECT to quantify activity in individual segments of bowel in inflammatory bowel disease patients. As SPECT imaging takes 30 minutes, peristalsis of the bowel could potentially cause problems, particularly false positive distal activity. We did not need to use drugs for bowel paralysis, because distal false positives were not seen. We would conclude therefore that at the imaging times used, the labelled cells are still intramucosal and have not yet passed into the lumen. Certainly at three hours luminal transit of activity is seen and is useful in distinguishing terminal ileal disease from a long sigmoid loop in the right iliac fossa. It is therefore critical that SPECT is performed early, possibly immediately after the one hour planar scan to completely avoid this potential problem.

The maximum activity detected in each segment was summated giving an overall disease activity score based on 'samples' throughout the length of the bowel. In the small bowel the maximum activity in distal, mid, and proximal segments was summated but could not be compared with histology. Our 'overall' SPECT score does not therefore directly quantify all of the uptake throughout the bowel as in faecal collection studies. As our gold standard assessment of disease activity was based, however, on serial biopsy samples taken from each bowel segment we applied a similar methodology for SPECT quantification.

It is, however, possible using SPECT to accurately measure all of the bowel activity by summation of all bowel activity in every slice. A thresholding technique can be used to define the edge of the bowel sections. Whether this adds any useful information compared with our sampling technique is being investigated in a separate study.

As the number of functioning radiolabelled white cells available for bowel uptake will vary between patients, some form of standardisation for this variable is needed. Standardisation to the injected dose of activity is not ideal because this may not be a true measure of available functioning white cell activity because of variations in cell labelling efficiency, cell damage, and peripheral white cell count. Standardisation to marrow activity seems the optimal method, as bone marrow activity is thought to largely represent physiological uptake of functioning cells by a process of 'margination'24 rather than a combination of margination and destruction of cells, which occurs in the liver and spleen. However, there is much still to be learned about marrow granulocyte kinetics. As 1.5-2 hours is comparatively early in the lifetime of a granulocyte it is probable that most marrow activity on early images results from margination of functioning cells rather than cell destruction. the high elution rate from other cells such as monocytes suggests that they make a very small contribution to marrow or bowel activity. As most labelled cells are granulocytes, purification of granulocytes before labelling with ${ }^{99 \mathrm{~m}} \mathrm{Tc}$ HMPAO is not necessary although it has recently been described. ${ }^{25}$ Marrow activity seems to offer the best available reference point, which is borne out by the close correlation between histology and SPECT score. 'Normalisation' of bowel segment activity to marrow activity was therefore used in this study. A reason for not normalising to splenic activity is that hyposplenism may occur in some inflammatory bowel disease patients. ${ }^{26}$

Transaxial slices permitted clear separation of both lumbar spine marrow activity and bowel segment activity in both colon (Fig 1), and in small bowel (Fig 4), so that the bowel and marrow activities were easily measured. Colonic images were represented as a homogenous circle, rather than a ring, which might have been expected on a transverse slice. This is because of the spatial resolution limit of the system, which is about $15 \mathrm{~mm}$. On later images intraluminal activity may be responsible.

SPECT quantification of bowel uptake of ${ }^{99 m}$ Tc HMPAO correlated well with the degree of inflammation in each segment of colon (Fig 2). It also permitted good separation of different histological grades because the SPECT score for each grade was highly significantly different. This method therefore offers the potential to measure changes in disease activity in different parts of the bowel in response to agents that may vary in their site of maximum delivery and action.

There was a range of SPECT scores for a given histological grade. This may in part reflect true variations in disease activity within each histological grade as well as sampling error, particularly in Crohn's disease. As expected, correlations between overall SPECT score and indirect measures of bowel inflammation such as erythrocyte sedimentation rate, $C$ reactive protein, and albumin were comparatively weak. For small bowel disease only serum albumin correlated with the SPECT score (Table II). A close correlation was seen, however, between the SPECT score and CDAI for disease in the colon as well as at all sites. The extreme variation in SPECT score for a given CDAI reflects the fact that the CDAI includes many components that are not caused by inflammation. As the type of colitis was often not known at the initial assessment, we used the CDAI for all patients, which may have been an additional source of variation. As this measure includes stool frequency and systemic manifestations of both diseases, however, this seemed reasonable and was supported by the greatly increased CDAI scores in the three ulcerative colitis patients who required emergency colectomy. Moreover, the CDAI has previously been applied as an overall disease activity measure in both forms of colitis. ${ }^{1}$ 
The three patients requiring colectomy had greatly increased total SPECT scores suggesting a method of detecting high risk patients in addition to the original indices described by Truelove and Witts. ${ }^{27}$

As the three dimensional distribution of activity is measured using SPECT, we have found that the pseudo 3D images of the involved bowel clearly show the position of involved segments (Fig 3).

A close correlation was seen between small bowel SPECT score and CDAI (Table II). Disease activity is more difficult to assess in the small bowel than in the colon because histological examination is difficult to obtain and symptoms may result from stricturing rather than inflammation. As white cell scans often showed small bowel inflammation despite normal $\mathrm{C}$ reactive protein and erythrocyte sedimentation rate, no correlation was shown between these indices and overall small bowel SPECT score (Table II). This has also been seen in other studies. ${ }^{21} 25$ In these particular cases therefore, ${ }^{99} \mathrm{~m}$ Tc HMPAO white cell scanning was important in detecting small bowel inflammation, which would not have been detected using routine inflammatory markers alone. SPECT scanning could clearly separate this small bowel activity from background activity thus providing the potential for accurate quantification (Fig 4). Other workers have detected asymptomatic small bowel inflammation, confirmed by histological examination, using ${ }^{99 \mathrm{~m}} \mathrm{Tc}$ HMPAO white cell scanning. ${ }^{25}$ That study showed a very close correlation between histological results and scan activity but less so with symptoms as some patients with positive histological results, and a positive scan had no symptoms. These were therefore not false positives. Whether such subclinical disease should be treated is open to question but it may be important in predicting relapse and in assessing the ability of therapeutic agents to completely abolish bowel inflammation as a separate endpoint to clinical remission.

There have, however, been some reports of non-specific activity usually beyond three hours but occasionally as early as one hour, ${ }^{28}$ which could lead to false positives. In our experience diffuse, very low grade activity is occasionally seen particularly in the right iliac fossa or central abdomen. The configuration of this uptake does not conform to any particular bowel segment and is therefore easily distinguished from bowel mucosal activity. We have seen that true small bowel inflammation has a very characteristic appearance (Fig 4). Other authors have also commented that non-specific activity is faint and is easily distinguished from true mucosal bowel uptake. 4

We conclude that once a definite histological diagnosis of inflammatory bowel disease has been made, that the severity of inflammation can be reliably assessed using ${ }^{99 \mathrm{~m}}$ Tc HMPAO planar and SPECT imaging. Both the small and large bowel can be assessed at the same time. Although planar and SPECT quantification were not directly compared in this study, other studies have shown the subjective nature of planar quantification, which may not be a problem when following up the progress of individual patients but would cause difficulties in population studies comparing different patients.

SPECT is therefore most useful in clinical research studies to objectively assess response to treatments rather than in the day to day management of patients for which planar scanning is currently acceptable. Although Almer's study ${ }^{29}$ showed no benefit from using a computerised technique compared with visual grading, Giaffer et $a l^{21}$ showed the superiority of their computerised method over planar imaging. Further experience of quantification techniques such as SPECT will be needed before the role of computerised quantification is clarified. Furthermore, ${ }^{99 \mathrm{~m} T c}$ HMPAO SPECT imaging is safe, widely available, and can provide serial measurements during treatment that accurately reflect the histological disease activity of inflammatory bowel disease.

We are grateful for the expert advice provided by $\mathrm{Dr} S \mathrm{H}$ Saverymuttu and the technical assistance provided by Miss A French. We are indebted to Amersham International for their encouragement and support. (M J Weldon was the Amersham International Gastroenterology Research Fellow.) A prelimiInternational Gastroenterology Research Fellow.) A preliminary oral presentation of this work was given to the British
Society of Gastroenterology in September 1992 (Gut 1992; 33 (suppl 2): S3)

1 Saverymuttu S, Camilleri M, Rees H, Lavender J, Hodgson $\mathrm{H}$, Chadwick V. Indium 111-granulocyte scanning in the assessment of disease extent and disease activity in inflammatory bowel disease. A comparison with colonoscopy, histology, and fecal indium 111-granulocyte excretion. Gastroenterology 1986; 90: 1121-8.

2 Saverymuttu S, Peters A, Lavender J, Pepys M, Hodgson $\mathrm{H}$, Chadwick V. Quantitative faecal indium-111 labelled leucocyte excretion in the assessment of disease in Crohn's disease. Gastroenterology. 1983; 85: 1333-9.

3 Peters AM, Danpure HJ, Osman S, Hawker RJ, Henderson $\mathrm{BL}$, Hodgson $\mathrm{H}$, et al. Clinical experience with $99 \mathrm{mTc}$ $\mathrm{BL}$, Hodgson $\mathrm{H}$, et al. Clinical experience with $99 \mathrm{mTc}-$ hexamethylpropylene-amineoxime for labelling leucoct

4 Lantto E, Jarvi K, Krekela I, Lantto T, Taavitsainen M, Vedenkangas $H$. Technetium $-99 \mathrm{~m}$ hexamethyl propylene amine oxine leucocytes in the assessment of disease activity in inflammatory bowel disease. Eur $\mathcal{F}$ Nucl Med 1992; 19: 14-8.

5 Becker W, Fischback W, Weppler M, Mosl B, Jacoby G, Borner W. Radiolabelled granulocytes in inflammatory bowel disease: diagnostic possibilities and clinical indications. Nucl Med Commun 1988; 9: 693-701.

6 DeNardo GL, Macey DJ, DeNardo SJ, Zhang CG, Custer TR. Quantitative SPECT of uptake of monoclonal antiTR. Quantitative SPECT of uptake of mont

7 Danpure H, Osman S, Carroll M. The development of a clinical protocol for the radiolabelling of mixed leucocytes with ${ }^{99} \mathrm{Tc}^{\mathrm{m}}$ hexamethyl propylene amine oxime. Nucl Med Commun 1988; 9: 465-75.

8 Chang L. A method for attenuation correction in radionuclide computed tomography. IEEE Trans Nucl Sci NS 1978; 25: 638-42.

9 Weldon MJ, Joseph AEA, Maxwell JD. Single photon emission computerised tomography (SPECT); a novel technique for imaging inflammatory bowel disease. Gut 1992; 33 (suppl 2): $\$ 3$.

10 Best W, Becktel J, Singleton J, Kern F. Development of a Crohn's disease activity index. National Co-operative

Crohn's disease study. Gastroenterology 1976; 70: 439-44.
1 Harvey R, Bradshaw J. A simple index of Crohn's disease activity. Lancet 1980 ; i: 514.

12 Andre C, Descos L, Vignal J. C-reactive protein level predicts Crohn's disease relapse in quiescent patients. Gut

13 Bartholomeusz FDL, Shearman DJC. Measurement of activity in Crohn's disease. $\mathcal{F}$ Gastroenterol Hepatol 1989; 4 : 81-94.

14 Teahan K, Bjarnason I. Comparison of leukocyte excretion and blood loss in inflammatory bowel disease of the bowel. Gut 1993; 34: 1535-8.

15 Stein P, Gray G, Gregory P, Anderson M, Goodwin D McDougall I. Location and activity of ulcerative and Crohn's colitis by indium-111 leukocyte scan Gastroenterology 1983; 84: 388-93.

16 Carpani M, Peters AM, Knight D, Shuttle AW, Lavender JP, Hodgson HJ. Indium-111 Whole body retention: a 
method for quantification of disease activity in inflammatory bowel disease. $₹ \mathrm{Nucl}$ Med 1992; 33: 756-62.

17 Peters AM. Imaging inflammation: current role of labelled autologous leukocytes [Editorial]. $\mathcal{f} \mathrm{Nucl} \mathrm{Med} \mathrm{1992;} \mathrm{33:}$ 65-7.

18 Peters AM, Roddie ME, Danpure HJ, Osman S, Zacharopoulos GP, George P, et al. 99Tcm-HMPAO labelled leucocytes: comparison with 111 In-tropolonate labelled granulocytes. Nucl Med Commun 1988; 9: 449-63.

19 Scholmerich J, Schmidt E, Schumichen C, Billmann P, Schmidt $H$, Gerok W. Scintigraphic assessment of bowel involvement and disease activity in Crohn's disease using technetium $99 \mathrm{~m}$-hexamethyl propylene amine oxine as leukocyte label. Gastroenterology 1988; amine oxine

20 Saverymuttu SH, Lavender JP, Hodgson HJ, Chadwick VS. Assessment of disease activity in inflammatory bowel disease: a new approach using 111 In granulocyte scanning. BMF 1983; 287: 1751-3.

21 Giaffer MH, Tindale WB, Senior S, Barber DC, Holdsworth CD. Quantification of disease activity in Crohn's disease by computer analysis of Tc-99M hexamethyl propylene amine oxime (HMPAO) labelled leucocyte images. Gut 1993; 34: 68-74.

22 Spinelli F, Milella M, Sara R, Banfi F, Vigorelli R, Possa M, et al. The $99 \mathrm{mTc}$-HMPAO leukocyte scan: an alternative to radiology and endoscopy in evaluating the extent and the activity of inflammatory bowel disease. $f \mathrm{Nucl}$ Biol Med 1991; 35: 82-7.

23 Weldon MJ, Saverymuttu SH, Finlayson CJ, Joseph AEA, Maxwell JD. Accurate assessment of colonic inflammatory bowel disease (IBD) using early $(1 \mathrm{hr})$ technetium (Tc99m) HMPAO white cell scanning. Gut 1993; 34 (suppl 1): S62.

24 Peters A. Granulocyte kinetics and methods of evaluating cell performance. Nucl Med Commun 1988; 9: 687-92.

25 Sciarretta G, Furno A, Mazzoni M, Basile CPM. Technetium-99m hexamethyl propylene amine oxime granulocyte scintigraphy in Crohn's disease: diagnostic and clinical relevance. Gut 1993; 34: 1364-9.

26 Palmer K, Sherriff S, Holdsworth C, Ryan F. Further experience of hyposplenism in inflammatory bowel disease. Qf Med 1981; 200: 463-71.

27 Truelove S, Witts L. Cortisone in ulcerative colitis: final report on a therapeutic trial. $B M F$ 1955; ii: 1041-4.

28 Gibson $P$, Lichtenstein M, Salehi N, Hebbard G, Andrews $J$. Value of positive technetium- $99 \mathrm{~m}$ leucocyte in predicting intestinal inflammation. Gut 1991; 32: 1502-7.

29 Almer S, Franzen L, Peters AM, Tjadermo M, Ekberg S, Granerus G, et al. Do technetium-99m hexamethylpropylene amine oxime-labeled leukocytes truly reflect the mucosal inflammation in patients with ulcerative colitis? Scand $\mathcal{F}$ Gastroenterol 1992; 27: 1031-8. 